\title{
Treating Obesity: Lifestyle, New Options in Pharmacotherapy, and the Obesogenic Environment
}

\author{
Coen D.A. Stehouwer ${ }^{a, b, c}$
}

Obesity is a chronic disease of pandemic proportions. Obesity reduces life expectancy, because it is associated with hypertension, dyslipidemia, type 2 diabetes, cardiovascular disease, and certain types of cancer. Additionally, it is associated with a severe course of coronavirus disease 2019 (COVID-19), although it is unclear to what extent that association is causal [1]. Obesity is also strongly associated with blaming (by the lay public and, alas, also by health care professionals), stigma, and reduced quality of life [1]. Thus, effective prevention and treatment of obesity are of paramount importance.

Lifestyle interventions focused on diet and exercise remain the cornerstone of treatment. However, a meta-analysis of behavior-based randomized controlled trials (67 studies, 22,065 participants) showed, on average, only modest weight loss after 12 to 18 months $(2.4 \mathrm{~kg}, 95 \%$ confidence interval (CI), 1.9 to 2.9) [2]. Additionally, weight loss, once achieved, is extremely difficult to maintain. A meta-analysis of behavior-based randomized controlled trials (25 studies, 2,949 participants who had achieved an initial weight loss of 5 to $10 \mathrm{~kg}$ ) showed a residual weight loss at 12 months of only $1.6 \mathrm{~kg}(95 \% \mathrm{CI}, 0.9$ to 2.3$)$ [3]. This should not come as a surprise, because the biological adaptations to weight loss with regard to energy expenditure, satiety, appetite and nutrient absorption are such that weight regain is strongly favored above maintenance of weight loss [4]. Although clearly some individuals can successfully maintain weight loss over the long term, variables that strongly predict such success have not been identified. Lifestyle interventions alone therefore are unsuccessful in many obese individuals. Additionally, adjunctive pharmacotherapy (with orlistat, phentermine-topiramate, or naltrexone-bupropion), over the past decades, has been shown to be at most modestly effective $[5,6]$. Moreover, some drugs have been withdrawn from the market because of safety concerns; among these are amphetamines (addiction), fenfluramine (cardiac toxicity) and lorcaser-

Manuscript submitted April 5, 2021, accepted April 12, 2021

Published online April 25, 2021

${ }^{a}$ CARIM School for Cardiovascular Diseases, Maastricht University, Maastricht, the Netherlands

${ }^{b}$ Department of Internal Medicine, Maastricht University Medical Centre, Maastricht, the Netherlands

${ }^{\mathrm{c}}$ Corresponding Author: Coen D.A. Stehouwer, Department of Internal Medicine, Maastricht University Medical Centre, Maastricht, the Netherlands. Email: cda.stehouwer@mumc.nl

doi: https://doi.org/10.14740/jem737 in (cancer risk) [6]. Although bariatric surgery can be an effective treatment of obesity, the procedure is invasive, costs of this treatment are high and availability on a global scale is limited.

In this context, glucagon-like peptide-1 (GLP-1) agonists appear to be a promising development. Liraglutide and semaglutide are GLP-1 agonists that have been approved for the treatment of type 2 diabetes in adults and for reducing the risk of cardiovascular events in people with type 2 diabetes and cardiovascular disease. These GLP-1 agonists are also associated with weight loss, presumably because they decrease appetite and thus energy intake. Semaglutide, in particular, has been shown to induce weight loss in people with type 2 diabetes and in adults with obesity in a phase 2 trial [7].

Recently, the results of the STEP-1 trial have been published, which evaluated semaglutide (2.4 mg subcutaneously, once weekly) in people with overweight or obesity (mean body mass index, $38 \mathrm{~kg} / \mathrm{m}^{2}$ ) and without diabetes [8]. After 68 weeks, the mean body weight change was $-14.9 \%$ in the semaglutide group as compared to $-2.4 \%$ in the placebo group. Weight loss of $5 \%$ or more was achieved by $86.4 \%$ versus $31.5 \%$, of $10 \%$ or more by $69.1 \%$ versus $12.0 \%$, and of $15 \%$ or more by $50.5 \%$ versus $4.9 \%$ - differences that were all highly statistically significant, as well as clinically relevant. Secondary endpoints included cardiovascular risk factors, physical function and quality of life, all of which improved significantly. As was to be expected, the main side effects were gastrointestinal in nature, especially nausea. These were transient in most people. Gallbladder-related disorders (especially cholelithiasis) were the most common severe side effect, consistent with previous reports on GLP-1 agonists and on rapid weight loss in general [8].

Limitations of the trial include its modest size $(1,961$ participants) and a participant population that was predominantly white $(75 \%)$ and female (74\%). The short follow-up (68 weeks) meant that long-term efficacy could not be demonstrated, whether during continuous drug administration or after stopping treatment. Additionally, cost-effectiveness also needs to be addressed [6]. Although semaglutide was also associated with improvement of cardiovascular risk factors, the study was not powered to address cardiovascular outcomes. A cardiovascular outcome trial of semaglutide in people with overweight or obesity and prior cardiovascular disease is ongoing (ClinicalTrials.gov number, NCT03574597). Although these limitations are important, semaglutide nevertheless appears a promising and welcome addition to the pharmacotherapy of overweight and obesity.

However, we should not lose sight of a fundamental truism: in the final analysis, weight gain and obesity are a matter 
of behavior. All behavior occurs through interactions between individuals and their environment, which, on a global scale, has become increasingly obesogenic. The obesogenicity of an environment has been defined as "the sum of influences that the surroundings, opportunities, or conditions of life have on promoting obesity in individuals or populations" [9]. For example, an individual's energy intake will be influenced by the availability, palatability and cost of food, which often tend to favor the intake of energy-dense and highly palatable foodstuffs. In turn, energy expenditure will be influenced by the physical design of the built environment, by land-use patterns and by transportation systems, all of which in general tend towards decreasing energy expenditure.

Attempts to stem the obesity pandemic through exclusively or even mostly focusing on an individual's own responsibility for his or her behavior disregard the strong effects of the environment on behavior. Such a strategy will, therefore, in the long run, not be successful.

What is needed is a societal strategy that focuses both on individuals, aiming at preventing weight gain or losing weight through improving lifestyle, if needed supported by pharmacotherapy, and the environment, aiming at a broad reduction of its obesogenic nature.

\section{Acknowledgments}

None to declare.

\section{Financial Disclosure}

None to declare.

\section{Conflict of Interest}

The author declares that he has no conflict of interest concerning this article.

\section{Author Contributions}

CDAS wrote and approved the final paper.

\section{Data Availability}

The author declares that data supporting the findings reported in this article are available within the article.

\section{References}

1. Stehouwer CDA. Observational research on severe COVID-19 in diabetes. Lancet Diabetes Endocrinol. 2021;9(2): 56-57.

2. LeBlanc ES, Patnode CD, Webber EM, Redmond N, Rushkin M, O'Connor EA. Behavioral and pharmacotherapy weight loss interventions to prevent obesity-related morbidity and mortality in adults: updated evidence report and systematic review for the US preventive services task force. JAMA. 2018;320(11):1172-1191.

3. Dombrowski SU, Knittle K, Avenell A, Araujo-Soares V, Sniehotta FF. Long term maintenance of weight loss with non-surgical interventions in obese adults: systematic review and meta-analyses of randomised controlled trials. BMJ. 2014;348:g2646.

4. Greenway FL. Physiological adaptations to weight loss and factors favouring weight regain. Int J Obes (Lond). 2015;39(8):1188-1196.

5. Bessesen DH, Van Gaal LF. Progress and challenges in anti-obesity pharmacotherapy. Lancet Diabetes Endocrinol. 2018;6(3):237-248.

6. Ingelfinger JR, Rosen CJ. STEP 1 for effective weight control - another first step? N Engl J Med. 2021;384(11):10661067.

7. O'Neil PM, Birkenfeld AL, McGowan B, Mosenzon O, Pedersen SD, Wharton S, Carson CG, et al. Efficacy and safety of semaglutide compared with liraglutide and placebo for weight loss in patients with obesity: a randomised, double-blind, placebo and active controlled, dose-ranging, phase 2 trial. Lancet. 2018;392(10148):637-649.

8. Wilding JPH, Batterham RL, Calanna S, Davies M, Van Gaal LF, Lingvay I, McGowan BM, et al. Once-weekly semaglutide in adults with overweight or obesity. N Engl J Med. 2021;384(11):989-1002.

9. Lake A, Townshend T. Obesogenic environments: exploring the built and food environments. J R Soc Promot Health. 2006;126(6):262-267. 